\title{
Characterization and Analysis of InGaAsSb Detectors
}

\author{
M. Nurul Abedin ${ }^{\mathrm{a}}$, Tamer F. Refaat ${ }^{\mathrm{b}}$, Ravindra P. Joshic, \\ Oleg V. Sulima ${ }^{\mathrm{d}}$, Michael Mauk ${ }^{\mathrm{d}}$ and Upendra N. Singh ${ }^{\mathrm{a}}$ \\ ${ }^{a}$ NASA Langley Research Center, Hampton, VA 23681 \\ ${ }^{\mathrm{b}} \mathrm{Science}$ and Technology Corporation, Hampton, VA 23666 \\ ${ }^{\mathrm{c}}$ Old Dominion University, Norfolk, VA 23529 \\ ${ }^{\mathrm{d}}$ AstroPower Incorporation, Newark, DE 19702
}

\begin{abstract}
Profiling of atmospheric $\mathrm{CO}_{2}$ at $2 \mu \mathrm{m}$ wavelength using the LIDAR technique, has recently gained interest. Although several detectors might be suitable for this application, an ideal device would have high gain, low noise and narrow spectral response peaking around the wavelength of interest. This increases the detector signal-to-noise ratio and minimizes the background signal, thereby increasing the device sensitivity and dynamic range. Detectors meeting the above idealized criteria are commercially unavailable for this particular wavelength. In this paper, the characterization and analysis of $\mathrm{Sb}$-based detectors for $2 \mu \mathrm{m}$ lidar applications are presented. The detectors were manufactured by AstroPower, Inc., with an InGaAsSb absorbing layer and AlGaAsSb passivating layer.

The characterization experiments included spectral response, current versus voltage and noise measurements. The effect of the detectors bias voltage and temperature on its performance, have been investigated as well. The detectors peak responsivity is located at the $2 \mu \mathrm{m}$ wavelength. Comparing three detector samples, an optimization of the spectral response around the $2 \mu \mathrm{m}$ wavelength, through a narrower spectral period was observed. Increasing the detector bias voltage enhances the device gain at the narrow spectral range, while cooling the device reduces the cut-off wavelength and lowers its noise. Noise-equivalent-power analysis results in a value as low as $4 \times 10^{-12} \mathrm{~W} / \mathrm{Hz}^{1 / 2}$ corresponding to $\mathrm{D}^{*}$ of $1 \times 10^{10} \mathrm{cmHz}^{1 / 2} / \mathrm{W}$, at $-1 \mathrm{~V}$ and $20{ }^{\circ} \mathrm{C}$. Discussions also include device operational physics and optimization guidelines, taking into account peculiarity of the Type II heterointerface and transport mechanisms under these conditions.
\end{abstract}

Keywords: IR Detectors, InGaAsSb, Characterization, Model

\section{INTRODUCTION}

Measurement of atmospheric carbon dioxide $\left(\mathrm{CO}_{2}\right)$ has gained recent interest. $\mathrm{CO}_{2}$ is one of the dominant greenhouse gases, that directly contributes to the global warming problem, but yet it has lot of uncertainties in term of its natural cycle, distribution and sources and sinks ${ }^{1}$. The differential absorption lidar (DIAL) is one of the powerful remote sensing techniques that might be suitable for profiling atmospheric $\mathrm{CO}_{2}$ with high accuracy ${ }^{2,3}$. Considering the recent advances in the development of the $2 \mu \mathrm{m}$ lasers ${ }^{3}$, Ismail et al simulated $\mathrm{CO}_{2}$ measurements using the DIAL technique at this wavelength ${ }^{4,5}$, while Ambrico et al compared the sensitivity of both 1.6 and $2 \mu \mathrm{m}$ DIAL measurements and emphasized the advantage of the $2 \mu \mathrm{m}$ wavelength ${ }^{2}$ and Taczak et al showed attempts for detecting $\mathrm{CO}_{2}$ using $2 \mu \mathrm{m}^{3}$.

Among different components of the DIAL system, the optical detector is of special interest. The detector converts the optical power of the lidar return signal into an electrical signal, thereby directly affecting the instrument sensitivity ${ }^{6}$. Being the dominant noise source in any lidar instrument, the detector affects the system minimum detectable signal and the corresponding minimum detectable concentration at a certain range ${ }^{7}$. These issues drive the need for a detector of narrow spectral bandwidth, high quantum efficiency and low noise. Small area quantum detectors such as avalanche photodiodes (APDs), are an attractive solution for lidar receivers due to their internal gain mechanism and relatively low noise. Simulation showed that for accurate $\mathrm{CO}_{2}$ DIAL measurements a detector noise-equivalent-power (NEP) in the 
range of $10^{-14} \mathrm{~W} / \mathrm{Hz}^{1 / 2}$, is necessary with $55 \%$ quantum efficiency and at least $300 \mu \mathrm{m}$ diameter of the active area ${ }^{5}$. Responsivity as high as $50 \mathrm{~A} / \mathrm{W}$, narrow spectral bandwidth and room temperature operation are further advantages of such device.

InGaAsSb is a promising semiconductor for developing high gain $2 \mu \mathrm{m}$ detectors. Several articles discussed the properties of this material and its application in APD fabrication using the separate absorption and multiplication (SAM) structure $^{8-16}$. Electron and hole impact ionization coefficients were evaluated and multiplication factors of 10-20 and 50100 were achieved at 296 and $78 \mathrm{~K}$, respectively, by Andreev et $\mathrm{al}^{8-10}$. The same group studied GaInAsSb/GaAlAsSb APDs fabricated using SAM structure, and examined the relation between the ionization coefficients and the excess noise factor'. Unlike silicon APDs, it was found that holes dominate the impact ionization process in this material structure $^{8}$. Spectral response peak shift toward longer wavelength with higher operating bias voltage was also observed in some devices ${ }^{11}$. Benoit et al studied similar structure and emphasis on the electric field constraints for fast and sensitive devices with dark current reduction ${ }^{12}$. Voronina et al studied the mobility of charge carriers in InGaAsSb and its dependence on both the doping concentration and the temperature ${ }^{13}$. Development of $p-n$, pin and SAM APD structures were discussed by Shellenbarger et al using the same material ${ }^{14-16}$. Spectral response profile dependence on frontal and backward illumination of the devices has been investigated by the same group. Backward illuminated p-n junction had narrower spectral band centered near $2 \mu \mathrm{m}$, which is an advantage for reducing the background signal while increasing the detector dynamic range.

In this paper, the development, characterization and modeling of InGaAsSb detectors are discussed for $2 \mu \mathrm{m} \mathrm{CO}_{2}$ DIAL applications. The detectors performance is compared to the system requirement. Suggestions on possible enhancement of the device performance, based on the numerical analysis, is pointed out.

\section{InGaAsSb DETECTOR STRUCTURE AND PROCESSING}

Figure 1 shows a schematic of the photodetector structure considered for this study. The detectors structure consists of a $2 \mu \mathrm{m}$ thick passivating $\mathrm{p}^{+}-\mathrm{Al}_{0.28} \mathrm{Ga}_{0.72} \mathrm{As}_{0.014} \mathrm{Sb}_{0.986}, \mathrm{Zn}$ doped layer, and a $5 \mu \mathrm{m}$ thick absorbing $\mathrm{n}$ InGaAsSb, Te doped layer with a thin $\mathrm{p}^{+}-\mathrm{In}_{0.15} \mathrm{Ga}_{0.85} \mathrm{As}_{0.17} \mathrm{Sb}_{0.83}, \mathrm{Zn}$ doped layer between them. The doping level of the absorbing InGaAsSb layer was nominally between 2 and $4 \times 10^{16} \mathrm{~cm}^{-3}$.

\begin{tabular}{|c|}
\hline $\mathrm{p}^{+}-\mathrm{AlGaAsSb}$ \\
\hline $\mathrm{p}^{+}$-InGaAsSb \\
\hline $\mathrm{n}$-InGaAsSb \\
\hline $\mathrm{n}-\mathrm{GaSb}$ \\
\hline
\end{tabular}

Figure 1 Schematic of the InGaAsSb detectors structure.

The epitaxial layers of III-V compound materials were grown on GaSb substrates. Liquid phase epitaxial growth was applied using a standard sliding graphite boat technique and several solid-composition were investigated. The low bandgap InGaAsSb material was used for the device active layer, while the high bandgap AlGaAsSb was used as a passivation or multiplication layer. The layers were grown on $500 \mu \mathrm{m}$ thick, chemically polished, (100) oriented, $\mathrm{n}$-type GaSb wafers doped to $3-5 \times 10^{17} \mathrm{~cm}^{-3}$ with tellurium. Starting melt composition and temperatures for the growth of the epitaxial layers were determined from published liquid phase equilibria data ${ }^{17}$ and experimentation. The metal components of the melt were added as high purity gallium, indium, and antimony shot pieces and aluminum wire. The arsenic was added as un-doped GaAs or InAs polycrystalline material. Prior to the growth, the metal components of the melts were heated to $700{ }^{\circ} \mathrm{C}$ for 15 hours under flowing hydrogen to de-oxidize the metallic melt components and outgas 
residual impurities. After cooling the melt 7 to $15{ }^{\circ} \mathrm{C}$ below the equilibrium temperature, the substrate was contacted with the melts at a cooling rate of $1{ }^{\circ} \mathrm{C} / \mathrm{min}$ to grow the device layers. Growth rates were typically $2-3 \mu \mathrm{m} / \mathrm{min}$. The composition of the epitaxial layers were determined by electron microprobe analysis using wavelength dispersive spectroscopy. The quality of the device layers was assessed using optical scanning electron microscopy. Good quality layers exhibited no cross-hatched pattern associated with lattice-mismatch of the substrate and epitaxial layers ${ }^{16}$.

Prototype mesa photodiodes with $200-300 \mu \mathrm{m}$ active area diameter $(400-\mu \mathrm{m}$ total area diameter) were formed using photolithography and chemical etching. Metallization for back n-type contacts was planar Au/Sn while front ptype contacts were annular $\mathrm{Au} / \mathrm{Zn}$. A spin-on, photosensitive polyimide layer was deposited and patterened prior to front contact deposition. This had several functions including planarizing the surface for the front contact deposition, providing insulation of the junction, and passivating the edges of the device area. After the photodiodes were formed, the substrate was diced into $1 \mathrm{~mm}^{2}$ pieces with a single device in the middle of each square. These were mounted to TO-18 headers using silver electrically conducting epoxy and wire-bonded from the bonding pad of the front contact to the header post.

\section{DEVICE CHARACTERIZATION}

Several prototype InGaAsSb/AlGaAsSb photodetectors were characterized in order to compare their performance with the requirement of the $\mathrm{CO}_{2}$ DIAL measurements. The characterization experiments included spectral response, dark current and noise measurements, and its variation with bias voltage and temperature. Figure 2 shows the experimental setup used to obtain these characteristics ${ }^{18}$. The setup is divided into an optical section and electrical section. The optical section is used to apply a uniform, monochromatic radiation onto the detector, with known intensity. The electrical section mainly measures the detector output, corresponding to a certain operating conditions, while maintaining these conditions.

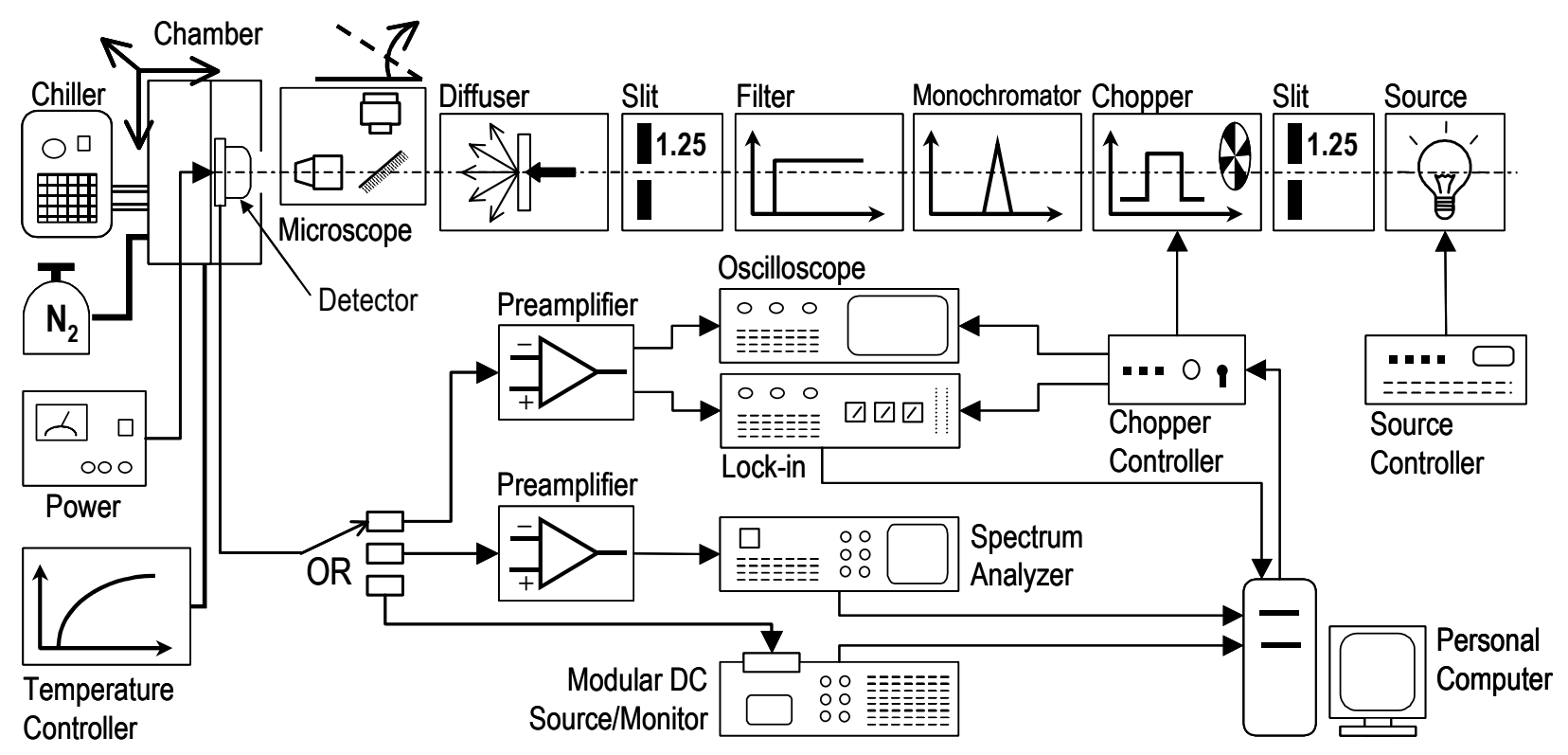

Figure 2 Experimental setup. 
The radiation source consists of a current controlled quartz halogen lamp, the output of which is modulated using an optical chopper. The chopping frequency is set to a prime number of $167 \mathrm{~Hz}$ to reduce the effect of pickup noise. A monochromator is used to separate the radiation into its spectral components with a $40 \mathrm{~nm}$ maximum resolution as set by the input/output slits and the grating $(1.25 \mathrm{~mm}$ input and output slits and 1200 Grove/Line grating). Higher order dispersion of the shorter wavelength is blocked using appropriate high-pass filters, while a diffuser is mounted at about $10 \mathrm{~cm}$ from the detector to insure radiation uniformity. An optical microscope was used to set the location of the optical axis and to fix the distance between the radiation source and the sensitive area of the detector. The radiation uniformity is estimated to be less than $1 \%$ along a $15 \mathrm{~mm}^{2}$ area at the detector location.

The detector output current is converted into voltage signal using the pre-amplifier (Stanford Research Systems; SR570), the output of which is applied to a lock-in amplifier (Optronic Laboratories, Inc.; OL 750-C), oscilloscope (Agilent; infiniium) or spectrum analyzer (Stanford Research Systems; SR785), for spectral response and noise measurements. For dark current measurements, a modular dc source/monitor (Hewlett Packard; 4142B) is connected directly to the detector. The chopper controller synchronizes the applied optical signal, if any, with the data acquisition device through the personal computer. To bias the detector, the pre-amplifier is used for voltages in the 0 to $4 \mathrm{~V}$ range while the external dc power supply is used for higher voltages. The detector is mounted inside a chamber that controls its temperature and provides mechanical support. The temperature is controlled using thermoelectric coolers and a thermistor, located as close as possible to the device. Detector temperature can be set between room temperature and -23 ${ }^{\circ} \mathrm{C}$ with $0.1{ }^{\circ} \mathrm{C}$ resolution. Water circulation through a chiller removes excess heat accumulation and nitrogen purging prevents water condensation and ice formation on the detector surface at lower temperatures. The mechanical mount allows detector alignment within $10 \mu \mathrm{m}$ resolution.

\subsection{Spectral Response Measurements}

The spectral response of the tested detectors were obtained using the substitution method, in which the calibration of a reference detector is transferred to the test detector ${ }^{19}$. This is done by comparing the output of both detectors resulting from a certain fixed radiation source at constant wavelength. Assuming $\mathfrak{I}$ is the intensity of the radiation source (in $\mathrm{W} / \mathrm{m}^{2}$ ), the reference detector output, $\mathrm{V}_{\mathrm{r}}$ will be given by

$\mathrm{V}_{\mathrm{r}}=\mathrm{A}_{\mathrm{r}} \cdot \mathfrak{R}_{\mathrm{r}} \cdot \mathfrak{I}$

where $A_{r}$ and $\mathfrak{R}_{r}$ are the reference detector sensitive area and responsivity (in $V / W$ ), respectively. Now, if the test detector were to be located at the same position with respect to the same source, its output $V_{t}$ will be given by

$\mathrm{V}_{\mathrm{t}}=\mathrm{G} \cdot \mathrm{A}_{\mathrm{t}} \cdot \Re_{\mathrm{t}} \cdot \mathfrak{I}$

where $G$ is the preamplifier gain and $A_{t}$ and $\mathfrak{R}_{t}$ are the test detector sensitive area and responsivity (in $A / W$ ), respectively. From equations (1) and (2), the test detector responsivity can be determined knowing the reference detector responsivity and the output of both detectors due to the same radiation source. In mathematical form

$\Re_{\mathrm{t}}=\frac{\mathrm{CF}}{\mathrm{G}} \cdot \frac{\mathrm{A}_{\mathrm{r}}}{\mathrm{A}_{\mathrm{t}}} \cdot \frac{\mathrm{V}_{\mathrm{t}}}{\mathrm{V}_{\mathrm{r}}} \cdot \Re_{\mathrm{r}}$

where the factor $\mathrm{CF}$ represent a correction factor, which accounts for any different condition between the reference and test detector measurements (ideally, $\mathrm{CF}=1$ ).

A $3 \times 3 \mathrm{~mm}^{2} \mathrm{PbS}$ calibrated reference detector was used to measure the spectral response of the tested detectors in the 1 to $2.4 \mu \mathrm{m}$ spectral range, with $40 \mathrm{~nm}$ spectral increments. Figure 3a shows the spectral response for two detector samples. Sample 1 indicated lower responsivity relative to sample 2. Responsivity enhancement in the 1.8 to $2.2 \mu \mathrm{m}$ spectral range was observed as emphasized in figure $3 \mathrm{~b}$, for three more samples. Responsivity reduction for wavelengths shorter than $1.8 \mu \mathrm{m}$ has the advantage of reducing the background signal which increase the detector dynamic range for the DIAL return signal. Besides, this might lower the restriction regarding the narrow band pass filter required with the DIAL detection ${ }^{7}$. Figure 4 shows sample 1 detector spectral response variation with temperature, obtained at $0 \mathrm{~V}$ bias voltage. Cooling down the detectors resulted in shifting the cutoff wavelength to shorter value, as expected due to the change in the energy bandgap ${ }^{20}$. Slight responsivity reduction in the 1.9 to $2.1 \mu \mathrm{m}$ range was observed at lower temperature. At shorter wavelength the responsivity of the detector remains constant. Lower temperature operation 
reduced the device noise. Figure 5 shows the spectral response variation with bias voltage for the same sample, obtained at about $6.5^{\circ} \mathrm{C}$. Increasing the applied voltage bias, increases the responsivity in the narrow spectral period (1.9 to 2.1 $\mu \mathrm{m})$ with slight shift in the cut-off wavelength. Higher bias operation was associated with increased device noise.

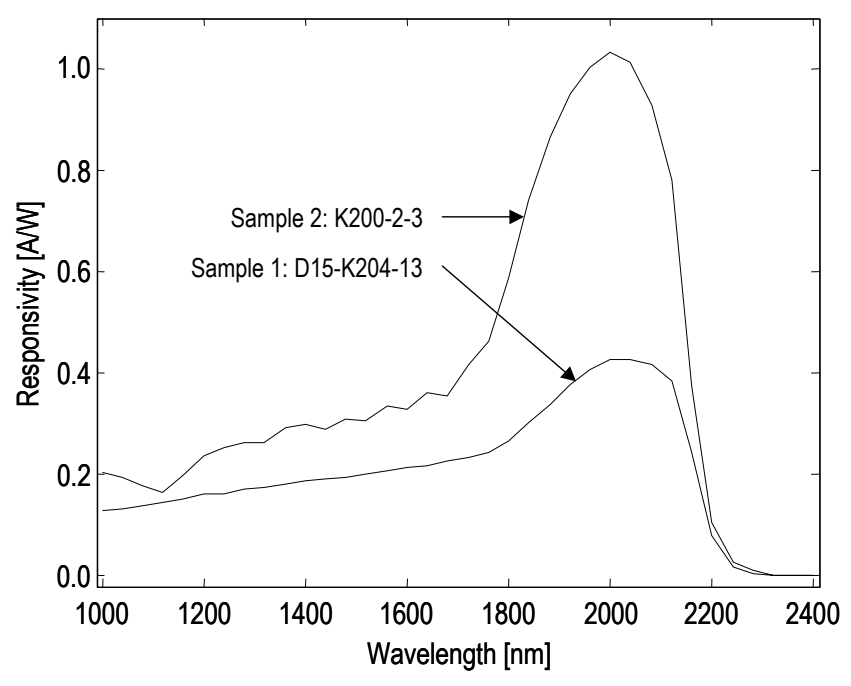

(a)

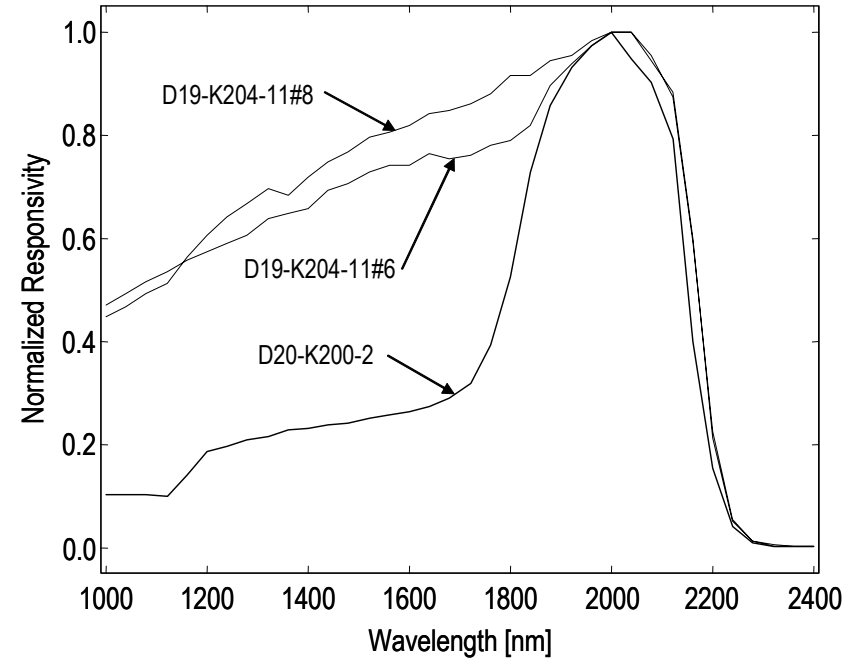

(b)

Figure 3 Spectral response for different detector samples obtained at (a) $0 \mathrm{~V}$ bias voltage and $20{ }^{\circ} \mathrm{C}$ temperature and (b) $1 \mathrm{~V}$ bias voltage and $20{ }^{\circ} \mathrm{C}$ temperature.

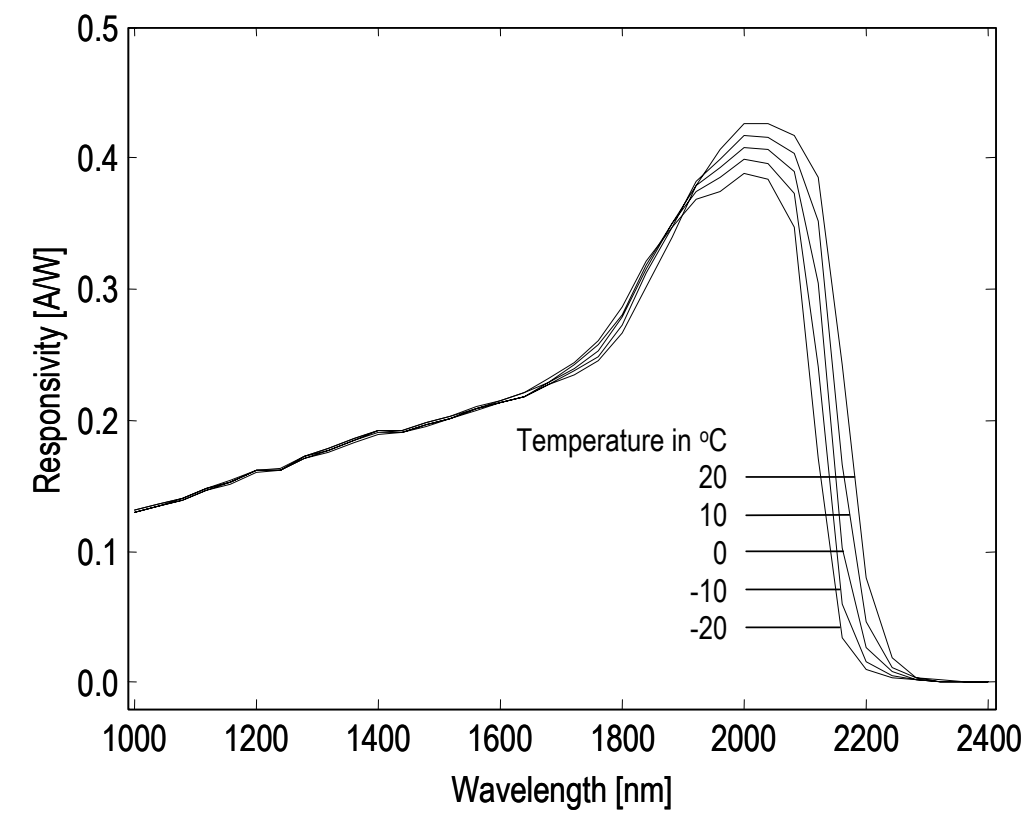

Figure 4 Spectral response variation with temperature, obtained at $0 \mathrm{~V}$ bias voltage for sample 1, D15-K204-13 pin structure 


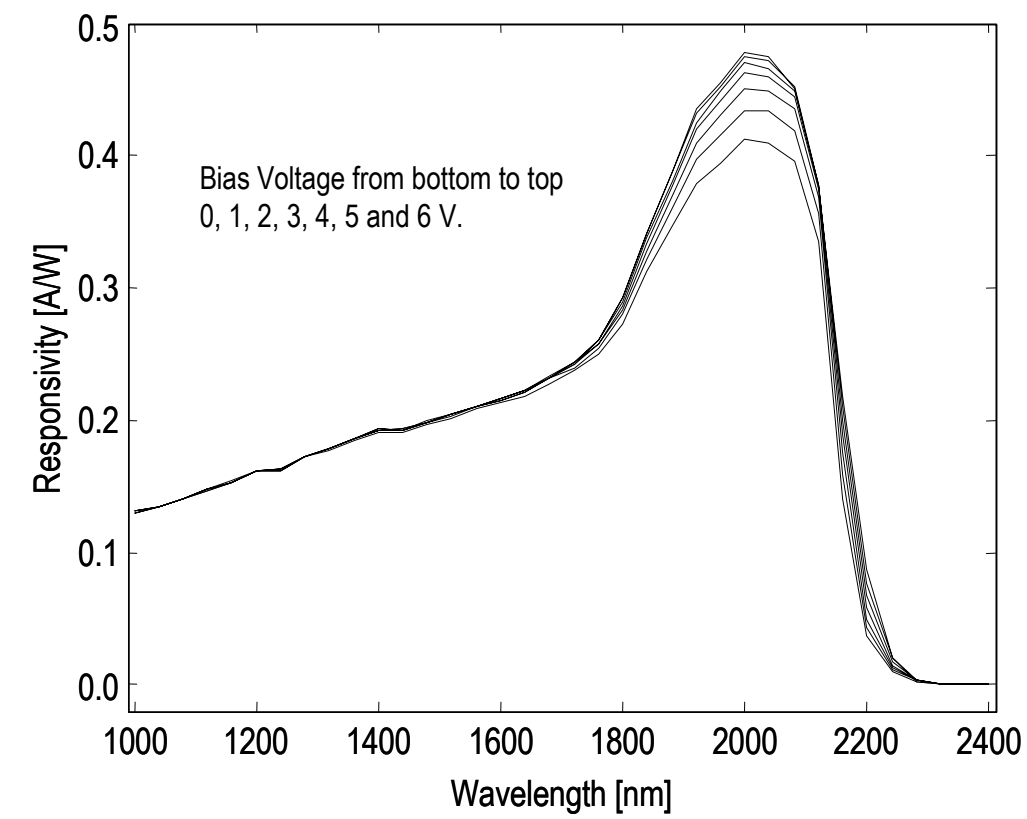

Figure 5 Spectral response variation with bias voltage, obtained at a temperature of $6.5^{\circ} \mathrm{C}$ for sample 1, D15-K204-13 pin structure.

\subsection{Dark Current Measurements}

Generally, the detector output current, $\mathrm{I}_{\mathrm{t}}$ can be expressed as

$\mathrm{I}_{\mathrm{t}}=\mathrm{I}_{\mathrm{o}}\left[\exp \left(\frac{\mathrm{qV}}{\mathrm{KT}}\right)-1\right]-\Re_{\mathrm{t}} \cdot \mathrm{P}$

where $I_{o}$ is the saturation dark current, $q$ is the electron charge, $V$ is the detector bias voltage, $\mathrm{K}$ is the Boltzmann's constant, $\mathrm{T}$ is the detector operating temperature and $\mathrm{P}$ in the power of the incident optical signal. The first term of equation (4) represent the dark current, while the second term represents the "useful" signal current. The dark current contribute to a dc offset at the preamplifier output. Ideally the dark current should be minimized to preserve the detector/ preamplifier dynamic range for the useful signal current. The variation of the dark current with temperature is of a special interest near breakdown, since it can be used to determine the breakdown mechanism, and identify avalanche gain if any ${ }^{14,16}$.

The dark current was measured at different temperatures as shown in figure 6. Although the device breakdown voltage was unidentified, measurements were nondestructive, as was examined by reproducing low voltage results before and after increasing the bias to near the breakdown level. The measurements were obtained using $100 \mathrm{mV}$ voltage step and 100 averaging. Increasing the bias voltage increases the dark current, which reduces the detector/ preamplifier dynamic range. The dark current can be also reduced by cooling down the detectors. The lower dark current of sample 1 might be due to slightly different doping concentration compared to sample 2 .

\subsection{Noise Measurements}

The noise voltage spectral density of the test detector was measured, at constant bias voltage and temperature, in conjunction with the preamplifier, using the spectrum analyzer. Measurements were obtained in the frequency band $1 \mathrm{~Hz}$ to $100 \mathrm{kHz}$ with $1 \mathrm{~Hz}$ normalization in dark conditions. Knowing the preamplifier gain, the noise voltage was then converted to noise current spectral density. The noise spectrum was averaged 1000 times with $50 \Omega$ termination, and the mean value was calculated in the operating frequency band. 


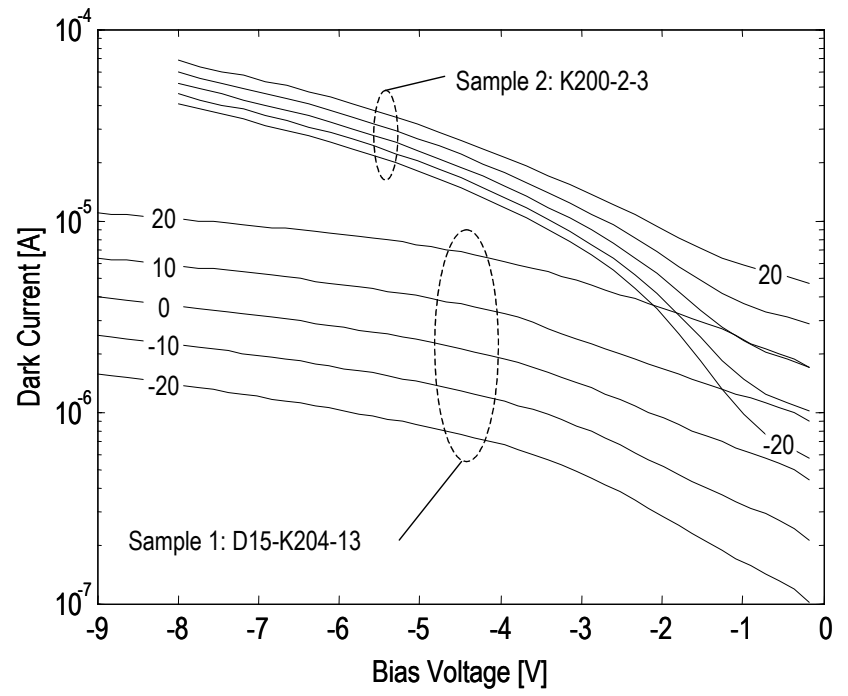

Figure 6 Dark current variation with bias voltage obtained at different temperatures for both samples.

In order to separate the detector noise current $\mathrm{I}_{\mathrm{n}}{ }^{\mathrm{d}}$, the preamplifier noise current, $\mathrm{I}_{\mathrm{n}}{ }^{\mathrm{a}}$ was measured associated with the setup with the detector replaced by a short circuit. The net detector noise current was then calculated by subtracting the amplifier and setup noise from the total noise current $I_{n}$ measured with the detector using the relation.

$\mathrm{I}_{\mathrm{n}}^{\mathrm{d}}=\sqrt{\left(\mathrm{I}_{\mathrm{n}}\right)^{2}-\left(\mathrm{I}_{\mathrm{n}}^{\mathrm{a}}\right)^{2}}$

For the InGaAsSb material structures under investigation, noise increases with increasing device temperature and bias voltage. Table 1 shows the noise current spectral density at $2 \mu \mathrm{m}$ with different operating conditions. The amount of the optical power incident on a detector that produces an output signal equal to the noise signal defines the NEP. For better detector comparison, the NEP is normalized to a bandwidth of $1 \mathrm{~Hz}$, and is given by ${ }^{18}$

$\mathrm{NEP}=\frac{\mathrm{I}_{\mathrm{n}}}{\Re}$

The detectivity of a detector $\left(D^{*}\right)$ is an efficient figure of merit when comparing detectors with different areas. The $\mathrm{D}^{*}$ is defined by the reciprocal of the NEP normalized to the detector sensitive area A, and is given by ${ }^{18}$

$\mathrm{D}^{*}=\frac{\sqrt{\mathrm{A}}}{\mathrm{NEP}}$

Measuring the detector noise currents, the calibration results were used to estimate its NEP and $\mathrm{D}^{*}$ as given in table 1 for the same calibration conditions. Although the table indicates similar noise level for these samples compared to similar devices reported in the literature, none of the samples matches the $\mathrm{CO}_{2}$ DIAL requirement for the NEP value of $2 \times 10^{-14} \mathrm{~W} / \mathrm{Hz}^{1 / 2}$.

Table 1 Detectors NEP and D* calculations corresponding to noise and responsivity measurements at $2 \mu \mathrm{m}$.

\begin{tabular}{|c|c|c|c|c|c|c|}
\hline $\begin{array}{c}\mathrm{T} \\
{ }^{0} \mathrm{C}\end{array}$ & $\begin{array}{l}\text { V } \\
\text { V }\end{array}$ & $\begin{array}{c}\mathfrak{R} \\
\mathbf{A} / \mathbf{W}\end{array}$ & $\begin{array}{c}\mathbf{I}_{\mathbf{n}} \\
\mathrm{A} / \mathbf{H z}^{1 / 2}\end{array}$ & $\begin{array}{c}\text { NEP } \\
\mathbf{W} / \mathbf{H z}^{1 / 2}\end{array}$ & $\begin{array}{c}D^{*} \\
\mathrm{cmHz}^{1 / 2} / \mathbf{W}\end{array}$ & $\begin{array}{l}\text { Detector/ } \\
\text { Diameter }\end{array}$ \\
\hline 20 & 0 & 0.4268 & $5.4 \times 10^{-12}$ & $12.6 \times 10^{-12}$ & $2.1 \times 10^{9}$ & D15-K204-13 \\
\hline-20 & 0 & 0.3879 & $5.3 \times 10^{-12}$ & $13.7 \times 10^{-12}$ & $1.9 \times 10^{9}$ & $300 \mu \mathrm{m}$ \\
\hline 20 & 0 & 1.0180 & $6.2 \times 10^{-12}$ & $6.1 \times 10^{-12}$ & $2.9 \times 10^{9}$ & K200-2-3 \\
\hline-20 & 0 & 1.0214 & $5.6 \times 10^{-12}$ & $5.4 \times 10^{-12}$ & $3.3 \times 10^{9}$ & $200 \mu \mathrm{m}$ \\
\hline 20 & -1 & 2.0226 & $8.1 \times 10^{-12}$ & $4.0 \times 10^{-12}$ & $5.5 \times 10^{9}$ & D20-K200-2 \\
\hline 20 & -4 & 2.0618 & $4.6 \times 10^{-11}$ & $2.2 \times 10^{-11}$ & $7.9 \times 10^{8}$ & $200 \mu \mathrm{m}$ \\
\hline
\end{tabular}




\section{DEVICE MODELING}

In order to simulate the response of a similar device structure ${ }^{16}$, the rate and transport equations of the excess charge carriers were considered under quasi-state conditions, after accounting for both photo-generation process in the InGaAsSb layer, and possible impact ionization process in the AlGaAsSb layer. The photo-generation rates were calculated considering Beer-Lambert attenuation after extracting the dependence of the absorption coefficient on the operating wavelength. A formula for the device multiplication factor was derived as a function of the bias voltage, since the impact ionization process is strongly reliant on the electric field. The electric field distribution along the device depth was obtained through a self-consistent solution of the coupled current continuity and Poisson's equations on the basis of the depletion approximation. Current flow at the hetero-interface was calculated assuming two electron fluxes incident from the opposite sides. The electron fluxes were calculated on the basis of the thermalized Fermi-Dirac distribution. In the numerical implementation, the Fermi-Dirac integral was approximated by a polynomial representation ${ }^{21}$.

The structure considered in this model is given in reference 16. Model parameters for the different materials are listed in Table 2 assuming room temperature operation ${ }^{16,22}$. The dependence of the absorption coefficient on wavelength is of particular interest for successful simulation of the absorption region. Although the absorption coefficients of InGaAsSb have been reported, none of the reports provide its value for the composition considered in this study, besides being out of the spectral range of interest ${ }^{23,24}$. Generally, the variations in the absorption coefficient are expected for wavelengths close to the band-edge due to the energy-dependent density of states and possible band-tailing effects due to the relatively high doping of the present devices. Given this uncertainty, the wavelength dependent absorption coefficients were estimated, as shown in Figure 7, by curve fitting the model theoretical predictions to previously published data for similar devices ${ }^{14}$. Figure 7 reveals nearly uniform absorption for wavelengths below $1.8 \mu \mathrm{m}$, with a slight peak around $2.0 \mu \mathrm{m}$, followed by sharp drop about $2.2 \mu \mathrm{m}$. The high wavelength trend is consistent with the data of Yacoubi et $\mathrm{al}^{23}$.

Table 2 Model parameters at $300 \mathrm{~K}$.

\begin{tabular}{|l|c|c|c|c|}
\hline & n-GaSb & n-InGaAsSb & n-AIGaAsSb & $\mathbf{p}^{+}$-AIGaAsSb \\
\hline Thickness $[\boldsymbol{\mu m}]$ & & 4 & 3 & 3 \\
\hline Doping Concentartion[ $\left[\mathbf{c m}^{-3}\right]$ & & $10^{17}$ & $10^{17}$ & $10^{18}$ \\
\hline Bandgap $[\mathbf{e V}]$ & & 0.53 & 1.3 & 1.3 \\
\hline Conduction Band Offset $[\mathbf{e V}]$ & 0.3257 & & & \\
\hline Conduction Band Offset $[\mathbf{e V}]$ & & 0.02 & & \\
\hline
\end{tabular}

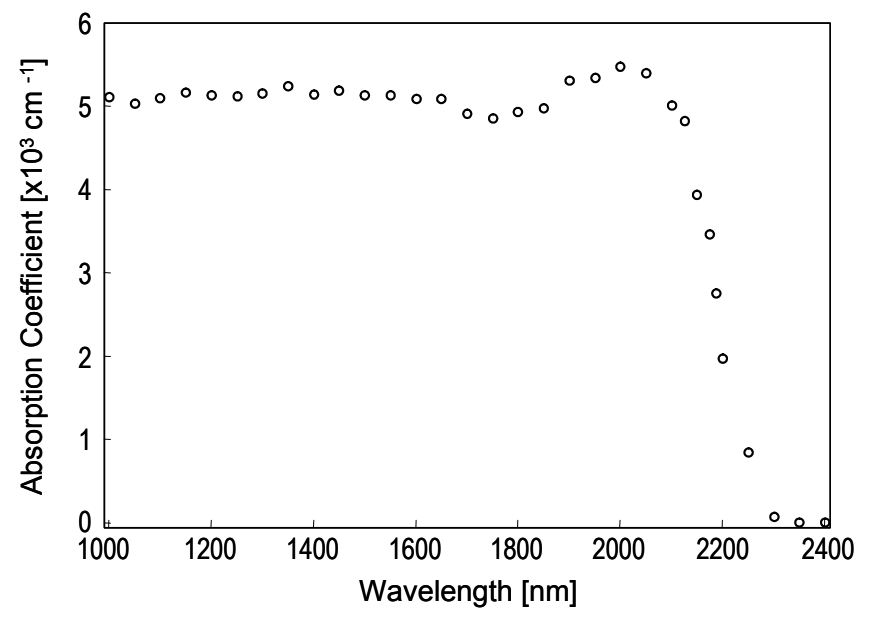

Figure 7 Estimated absorption coefficient as a function of wavelength for InGaAsSb in the 1 to $2.4 \mu \mathrm{m}$ spectral period. 
Computations of the internal field profile and the potential distribution among the different interfaces were carried out. Results of these calculations in the form of energy band diagram along the device depth are shown in figure 8 . The calculations assume room temperature operation of $300 \mathrm{~K}$, and presented at zero and $0.4 \mathrm{~V}$ bias voltage. The plots of figure 8 reveal several interesting features. First, due to the high doping used in the InGaAsSb, the depletion regions in the absorbing layer are relatively small, which decrease the efficiency of the transport and the sweep-out of the photogenerated charge carriers while increase their recombination probability. Second, the electric fields at the GaSbInGaAsSb and the AlGaAsSb p-n junctions do not inter-penetrate. This minimal overlap implies that electric fields within the bulk of the $\mathrm{n}-\mathrm{AlGaAsSb}$ region, that connects the absorbing region to the $\mathrm{p}$-AlGaAsSb collector, are low. This does not bode well for efficient photo-carrier transport and collection in such a photodetector. Third, by increasing the bias voltage, the additional drop occurs almost entirely over the AlGaAsSb p-n junction while the absorption layer remains partially depleted. While this increases the probability of the impact ionization in the multiplication region, the drift within the absorption region remains inefficient. Finally, high potential barriers at the InGaAsSb-AlGaAsSb heterointerface and the AlGaAsSb junction exist. The former barrier opposes the flow of the photo-generated holes while the latter opposes the electrons supplied from the external circuit.

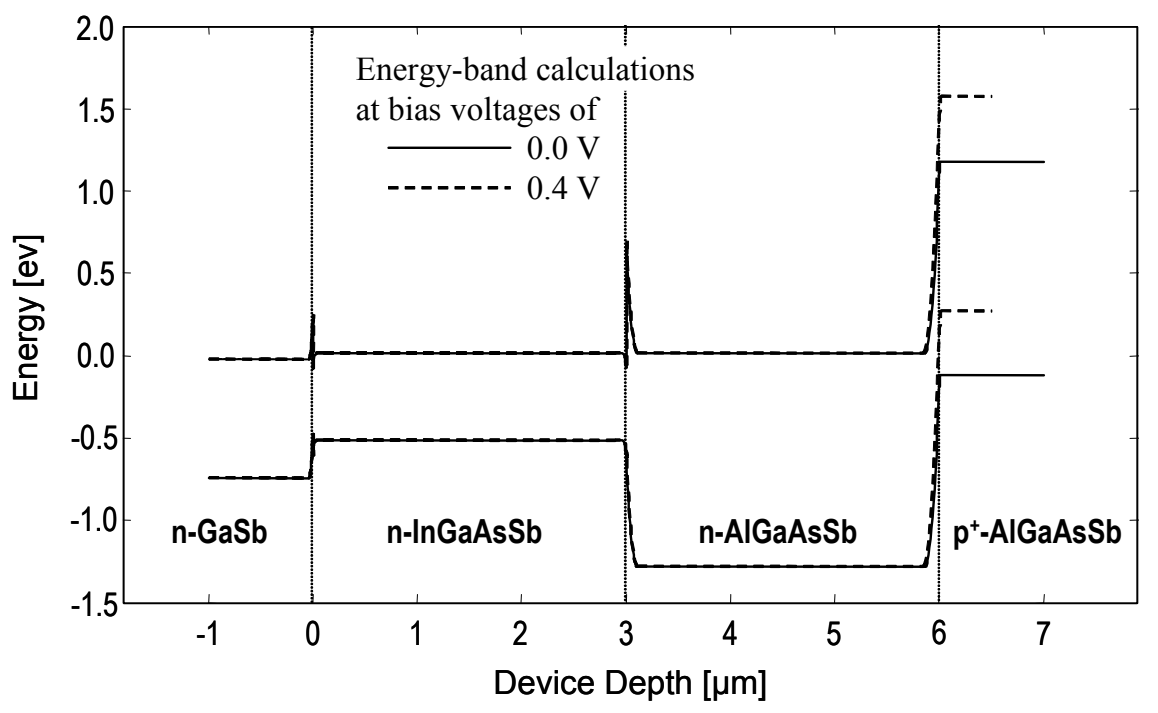

Figure 8 Energy band diagram calculation for InGaAsSb/AlGaAsSb SAM APD structure at two bias voltages.

Improving the device performance would require the reduction in the doping concentration in the $\mathrm{n}$-InGaAsSb absorption region. This would increase the depletion layer width and decrease carrier recombination, which leads to a faster and more efficient charge carrier drift to gain the maximum kinetic energy required for the following multiplication region. Lower doping would also increase the internal resistance which leads to lower dark current and noise. Reducing the doping concentration in the $\mathrm{n}-\mathrm{AlGaAsSb}$ region multiplication region, to nearly intrinsic level would reduce the valence band offset between the InGaAsSb-AlGaAsSb regions. This would reduce the barrier for the hole transport by pushing up the AlGaAsSb energy band. The electric field within the multiplication region would increase, which will increase the impact ionization probability in the p-n junction. Besides, lowering the doping in this layer would considerably reduce the tunneling current across the $\mathrm{p}-\mathrm{n}$ junction. Finally, reducing the width of AlGaAsSb region itself would cause the electric field in the n-p junction to penetrate deeper into the InGaAsSb region. The width reduction would lower the carrier transit time over the impact ionization zone. This will increase the frequency response and speed of the device while reducing the impact ionization noise. Optimization of the alloy composition to enhance the ratio of the impact ionization coefficients while remaining within the $2 \mu \mathrm{m}$ wavelength regime would dramatically enhance the device performance and will be the subject of future study. 


\section{CONCLUSION}

InGaAsSb is a promising material for developing high gain $2 \mu \mathrm{m}$ detectors. Being a quaternary structure, the detector characteristics could be tailored to match specific applications such as remote sensing of atmospheric $\mathrm{CO}_{2}$. Prototype detectors were fabricated using this material with AlGaAsSb layers on $\mathrm{GaSb}$ substrates. Detector characterization was discussed and included spectral response, current-voltage and noise measurements. Noise equivalent power and detectivity calculations were presented. By controlling the detector structure, narrow spectral periods were achieved between 1.9 and $2.1 \mu \mathrm{m}$, which has the advantage of dark current reduction. Cut-off wavelength shifts to lower value by cooling down the device. Lower temperature operation had the advantage of dark current and noise reduction. Increasing the bias voltage leads to a slight increase in the responsivity and is associated with increase in the device dark current and noise.

Improvement in the device performance was investigated by modeling the detector structure. The model concluded that for better characteristics the both the doping levels and the layer widths of the absorption and multiplication regions should be reduced. Future investigation is required by constructing prototype devices with the suggested modification.

\section{Acknowledgment}

This effort is part of the Low Risk Reduction Program funded by NASA's Earth Science Technology Office and the Enabling concepts and Technology Program within NASA's Aerospace Technology Enterprise. The authors would like to thank Frank Peri for his support.

\section{REFERENCES}

1. J. Kaiser and K. Schmidt, "Coming to grips with the world's greenhouse gases", Science, 281, 504-506, 1998.

2. P. Ambrico, A. Amodeo, P. Girolamo and N. Spinelli, "Sensitivity analysis of differential absorption lidar measurements in the mid-infrared region”, Applied Optics, 39(36), 6847-6865, 2000.

3. T. Taczak and D. Killinger, "Development of a tunable, narrow-linewidth, cw $2.066 \mu \mathrm{m}$ Ho:YLF laser for remote sensing of atmospheric $\mathrm{CO}_{2}$ and $\mathrm{H}_{2} \mathrm{O}$ ", Applied Optics, 37(36), 8460-8476, 1998.

4. S. Ismail, E. Browell, J. Barnes, G. Koch, J. Hair and U. Singh, "Studies toward the development of a space-based $2-\mu \mathrm{m}$ lidar system for profiling atmospheric $\mathrm{CO}_{2}$ ", International Geoscience and Remote Sensing Society Symposium, IGARSS2001, Sydney, Australia, July 2001.

5. S. Ismail, G. Koch, E. Browell, U. Singh, N. Abedin, J. Yu and J. Hair, "A 2-micron DIAL system for profiling atmospheric $\mathrm{CO}_{2}$ ", Lidar Remote Sensing in Atmospheric and Remote Sciences, $21^{\text {st }}$ International Laser Radar Conference, Quebec City, Canada, 2002.

6. D. Killinger and N. Menyuk, "Laser remote sensing of the atmosphere", Science, 235, 37-45, 1987.

7. R. Measure, Laser Remote Sensing: Fundamental and Applications, John Wiley \& Sons, Inc., 1984.

8. I. A. Andreev, M. P. Mikhailava, S. V. Mel'nikov, Yu. P Smorchkova and Yu. P. Yakovlev, "Avalanche multiplication and ionization coefficients of GaInAsSb", Soviet Physics-Semiconductor, 25(8), 861-865, 1991.

9. M. P. Mikhailova, I. A. Andreev, A. N. Baranov, S. V. Mel'nikov, Y. P. Smortchkova and Y. P. Yakovlev, "Lownoise GaInAsSb/GaAlAsSb SAM avalanche photodiode in the 1.6-2.5 $\mu \mathrm{m}$ spectral range", Proceeding of SPIE, 1580, 308-312, 1991.

10. I. A. Andreev, M. A. Afrailov, A. N. Baranov, M. A. Mirsagatov, M. P. Mikhailova and Yu. P. Yakovlev, "Avalanche multiplication in photodiode structures using GaInAsSb solid solutions", Soviet Technical Physics Letters, 13(4), 199-201, 1987.

11. I. A. Andreev, M. A. Afrailov, A. N. Baranov M. A. Mirsagatov, M. P. Mikhailov and Yu. P. Yakovlev, "GaInAsSb/GaAlAsSb avalanche photodiode with separate absorption and multiplication regions", Soviet Technical Physics Letters, 14, 435-437, 1988. 
12. J. Benoit, M. Boulou, G. Soulage, A. Joullie and H. Mani, "Performance evaluation of GaAlAsSb/GaInAsSb SAMAPDs for high bit rate transmission in the $2.5 \mu \mathrm{m}$ wavelength region", Journal of Optical Communication, 9(2), 55 $58,1988$.

13. T. Voronina, B. Dzhurtanov, T. Lagunova and Y. Yakovlev, "Behavior of impurities of p-type GaInSbAs solid solutions", Soviet Physics-Semiconductor, 25(2), 171-173, 1991.

14. Z. A. Shellenbarger, M. G. Mauk, M. I. Gottfried, J. D. Lesko and L. C. DiNetta, "GaInAsSb and InAsSbP photodetectors for mid-infrared wavelengths", Proceeding of SPIE, 2999, 25-33, 1997.

15. Z. A. Shellenbarger, M. G. Mauk, J. Cox, J. South, J. Lesko, P. Sims, M. Jhabvala and M. Fortin, "Recent progress in GaInAsSb and InAsSbP photodetectors for mid-infrared wavelengths", Proceeding of SPIE, 3287, 138-145, 1998.

16. Z. A. Shellenbarger, M. G. Mauk, J. Cox, J. South, J. Lesko, P. Sims, L. DiNetta, "GaInAsSb and InAsSbP photodetectors for mid-infrared wavelengths", Proceeding of SPIE, 3379, 354-360, 1998.

17. M. Astles, H. Hill, A. J. Williams, P. J. Wright and M. L. Young, "Studies of the $\mathrm{Ga}_{1-\mathrm{x}} \mathrm{In}_{\mathrm{x}} A \mathrm{As}_{1-\mathrm{y}} \mathrm{Sb}_{\mathrm{y}}$ Quaternary alloy system I. Liquid-phase epitaxial growth and assessment", Journal of Electronic Materials, 15(1), 41, 1986.

18. T. Refaat, N. Abedin, G. Koch and U. Singh, "InGaAsSb detectors' characterization for $2-\mu \mathrm{m} \mathrm{CO}_{2}$ lidar/DIAL applications", NASA Technical Publication, NASA/TP-2003-212140, 2003.

19. Thomas C. Larason, Sally S. Bruce and Albert C. Parr, "Spectroradiometric detector measurements: Part IUltraviolet detectors and Part II- Visible to near-infrared detectors", NIST Special Publication, 250-41, 1998.

20. T. Yuan, Z. Baolin, J. Yixin, Z. Tianming, L. Shuwe, N. Yongqiang, Y. Jinshan, J. Hong and Y. Guang, "Calculation on relation of energy bandgap to composition and temperature for $\mathrm{Ga}_{\mathrm{x}} \mathrm{In}_{1-\mathrm{x}} \mathrm{As}_{1-\mathrm{y}} \mathrm{Sb}_{\mathrm{y}}$ ", Rare Metals, 15(3), 172-178, 1996.

21. S. A. Wong, S. P. McAlister and Z. M. Li, "A comparison of some approximations for the Fermi-Dirac integral of order 1/2", Solid-State Electron, 37(1), 61-64, 1994.

22. A. Y. Polyakov, A. G. Milnes, A. V. Govorkov, L. V. Druzhinina, I. V. Tunitskaya, and N. B. Smirnov, "Band offsets in hetrojunctions of InGaAsSb/AlGaAsSb", Solid-State Electron, 38(10), 525-529, 1994.

23. N. Yacoubi, A. Hafaiedh and A. Joullie, "Determination of the optical and thermal properties of semicoductors with the photothermal method", Applied Optics 33(30), 7171-7174, 1994.

24. J. C. DeWinter, M. A. Pollack, A. K. Strivastava and J. L. N. Zyskind, "Liquid phase epitaxial GaInAsSb lattice matched to (100) GaSb over the 1.71 to $2.33 \mu \mathrm{m}$ wavelength range", Journal of Electronic Materials, 14(6), 729$747,1985$. 